\title{
Aceitação de preparações e sua associação com os sintomas decorrentes do tratamento de câncer em pacientes de uma clínica especializada
}

\section{Food acceptance and its association with cancer treatments symptoms in patients of a specialized clinic}

\author{
Bárbara Nogueira Palmieri' ${ }^{1}$, Eloisa Massaine Moulatlet ${ }^{1}$, \\ Lia Kanae Okita Buschinelli², Maria Elisabeth Machado Pinto-e-Silva ${ }^{3}$
}

\begin{abstract}
Resumo
O câncer e seus tratamentos desencadeiam alterações metabólicas e efeitos colaterais que afetam o estado nutricional do paciente e a aceitabilidade dos alimentos. O presente estudo avaliou a aceitação de preparações e sua associação aos sintomas decorrentes do tratamento em pacientes de uma clínica especializada em tratamento oncológico. Trata-se de um estudo transversal, descritivo e quantitativo. Coletaram-se dados clínicos de pacientes oncológicos, os quais avaliaram três preparações utilizando escala hedônica de cinco pontos. A aceitação das preparações, com grau de rigidez de 60\%, foi para os valores $\geq 4$ (gostei e gostei muito). Para análise da aceitação e relação entre as variáveis, foram utilizados os testes de análise de variância e de Tukey, com p<0,05. Participaram 23 pacientes, 87,0\% do sexo feminino e 65,2\% com excesso de peso. Cânceres de mama $(56,5 \%)$ e de pulmão $(17,4 \%)$ foram os mais frequentes, predominando o tratamento quimioterápico atual $(52,2 \%)$ e a presença de desconfortos gastrintestinais $(61,0 \%)$. Houve associação estatisticamente significante entre quimioterapia atual e disgeusia $(p<0,010)$, saciedade precoce e avaliação global do bolo de gengibre com linhaça $(p=0,049)$, xerostomia e textura do creme Chocolícia $(p=0,050)$ e disgeusia e avaliação global da mesma preparação $(p=0,045)$. Todas as preparações desenvolvidas foram bem aceitas, sendo a disgeusia o sintoma que apresentou alteração na aceitação.
\end{abstract}

Palavras-chave: neoplasias; quimioterapia; estado nutricional; dieta.

\begin{abstract}
Cancer and its treatments cause metabolic alterations and side effects that affect patients' nutritional status and food acceptability. This study evaluated acceptance of culinary preparations and its association with symptoms caused by cancer treatments in patients from a clinic specialized in oncologic treatment. This is a transversal, descriptive and quantitative study. Patients had their clinical data collected and evaluated three culinary preparations using the five-point hedonic scale. Preparations were considered as accepted when at least $60 \%$ of responses were $\geq 4$ (liked and liked very much). For the analysis of acceptance and relation between variables, the analysis of variance and Tukey's tests were used, considering $p<0.05$. Twenty-three patients participated in the study, $87.0 \%$ were female and $65.2 \%$ were overweight. Breast (56.5\%) and lung $(17.4 \%)$ cancers were the most prevalent diagnosis. Most participants were under chemotherapy treatment (52.2\%) and reported gastrointestinal discomfort (61.0\%). There was statistically significant association between chemotherapy and
\end{abstract}

Trabalho realizado no Instituto Paulista de Cancerologia (IPC) - São Paulo (SP), Brasil.

${ }^{1}$ Graduanda do curso de Nutrição da Faculdade de Saúde Pública da Universidade de São Paulo (USP) - São Paulo (SP), Brasil.

${ }^{2}$ Nutricionista com Especialização em Oncologia pelo Hospital Israelita Albert Einstein; Nutricionista do IPC - São Paulo (SP), Brasil.

${ }^{3}$ Doutora em Saúde Pública pela Faculdade de Saúde Pública da USP; Docente do curso de graduação em Nutrição da USP - São Paulo (SP), Brasil.

Endereço para correspondência: Maria Elisabeth Machado Pinto-e-Silva - Avenida Doutor Arnaldo, 715 - Cerqueira César - CEP: 01246-904 - São Paulo

(SP), Brasil -E-mail: mmachado@usp.br

Fonte de financiamento: nenhuma.

Conflito de interesse: nada a declarar. 
dysgeusia $(p<0.01)$, early satiety and global evaluation of ginger cake with flaxseed $(p=0.049)$, xerostomia and texture of Chocolícia cream $(p=0.050)$ and dysgeusia and global evaluation of the same preparation $(p=0.045)$. All the recipes developed were well accepted, and dysgeusia was the symptom that interfered the most in its acceptance.

Keywords: neoplasms; drug therapy; nutritional status; diet.

\section{INTRODUÇÃO}

Câncer ou neoplasia maligna são termos utilizados para classificar um conjunto de doenças que têm em comum o crescimento desordenado, rápido e incontrolável de células, com a capacidade de invadir tecidos e órgãos e se espalhar para outras regiões do corpo, originando metástase ${ }^{1}$. A Agência Internacional para Pesquisa em Câncer (IARC) estimou a ocorrência de 12,4 milhões de novos casos e 7,6 milhões de óbitos por câncer no mundo, em $2008^{2}$. No Brasil, as estimativas para 2010 e 2011 apontam a incidência de 489.270 casos, prevalecendo os cânceres de próstata e pulmão no gênero masculino e os de mama e colo do útero no feminino, excluindo o câncer de pele do tipo não melanoma em ambos os sexos ${ }^{3}$.

Além da fisiopatologia da doença se caracterizar pelo desencadeamento de alterações metabólicas de grande magnitude, as modalidades de tratamento existentes podem resultar em efeitos colaterais que afetam o estado nutricional do paciente 4 . Cirurgia, radioterapia, quimioterapia ou a combinação dos mesmos podem causar desde dor e constipação até náuseas, vômitos, mucosites e falta de apetite, contribuindo para a redução da ingestão alimentar e consequente desnutrição $0^{4-6}$. Ademais, quanto mais agressivo o tratamento, mais progressiva é a desnutrição, resultando em diminuição da qualidade de vida e piora do estado geral. Assim, os objetivos da terapia nutricional são: prevenir e tratar a desnutrição, garantindo a oferta adequada de nutrientes para minimizar o catabolismo proteico e a perda nitrogenada; manter a atividade do sistema imune; melhorar a qualidade de vida; reduzir o número de complicações provenientes dos tratamentos e prevenir a interrupção dos mesmos ${ }^{4,6}$.

A falta de apetite é um sintoma comum em pacientes oncológicos, inicialmente relacionada ao processo natural da doença e, posteriormente, ao crescimento tumoral, à presença de metástases e aos tratamentos ${ }^{7}$. Saciedade precoce, disgeusia e xerostomia também são sintomas frequentes que podem surgir no decorrer do tratamento de câncer ${ }^{6}$. Na terapia nutricional, a via oral de alimentação deve ser sempre priorizada e, caso a aceitação da dieta seja inferior a $60 \%$ das necessidades, é preciso aumentar as densidades calórica e proteica por meio de alimentos calóricos e nutritivos ou de suplementos alimentares industrializados ${ }^{7}$. Sabe-se, porém, que a aceitabilidade e a palatabilidade dos suplementos são fatores fundamentais para a adesão ao tratamento, e o incômodo gerado pela oferta do mesmo sabor várias vezes ao dia diminui sua aceitação ${ }^{4}$.
Portanto, sugerir preparações apropriadas às condições atuais do paciente utilizando ou não suplementos alimentares como ingrediente é uma atribuição importante do nutricionista.

Náuseas e vômitos são outros sintomas possíveis em pacientes oncológicos, podendo ser decorrentes de tratamentos como quimioterapia; radioterapia das regiões abdominal, pélvica e de corpo inteiro; uso de medicamentos; desequilíbrios hidroeletrolíticos; mudanças de paladar e odor e obstrução intestinal ${ }^{8}$. Esses são os sintomas mais frequentes da quimioterapia, cuja emetogenicidade depende de características do paciente e do fármaco, de fatores psicológicos, da presença de desordens de motilidade gastrintestinal, entre outros. As náuseas e os vômitos atingem entre 70 e $80 \%$ dos pacientes que recebem quimioterapia e influenciam no estado nutricional, principalmente devido à diminuição da ingestão alimentar ${ }^{9}$. Orientações nutricionais representam um importante tratamento não farmacológico de tais sintomas, com o objetivo de preveni-los e minimizá-los. Nesse caminho, estudos sugerem o benefício do gengibre para controle de náuseas e vômitos induzidos pela quimioterapia ${ }^{10,11}$.

Cerca de 30\% dos pacientes oncológicos que não recebem opioides e entre 60 e 70\% dos que recebem esse tipo de medicamento apresentam constipação intestinal. Anestésicos e alguns quimioterápicos também podem ser responsáveis pelo sintoma, além de causas do próprio câncer e daquelas associadas ao seu tratamento, efeitos metabólicos e fatores extrínsecos à doença. $\mathrm{O}$ controle da constipação é dificultado devido à baixa ingestão de alimentos e líquidos, além de náuseas, vômitos e saciedade precoce, que podem estar associados, portanto, a intervenção nutricional é um recurso importante para minimizar este sintoma e prevenir desnutrição e desidratação, além de reduzir a necessidade do uso de métodos invasivos e desconfortáveis. A prevenção e o tratamento da constipação incluem ingestão adequada de fibras, cuja recomendação é de 25 a $35 \mathrm{~g} /$ dia para indivíduos com mais de 20 anos e de 10 a $13 \mathrm{~g}$ por $1.000 \mathrm{kcal}$ para idosos. As fibras devem ser preferencialmente ingeridas a partir de alimentos e seu consumo deve estar associado à adequada ingestão de líquidos ${ }^{12}$.

Devido à alteração na percepção do sabor e aos déficits na gustação (disgeusia), xerostomia e outros sintomas, além das complicações causadas pelo câncer e seus tratamentos ${ }^{13}$, é responsabilidade do nutricionista alterar a dieta de modo a torná-la atrativa e palatável ${ }^{4}$. Estudos realizados em uma clínica especializada em tratamento oncológico durante 2010 
revelaram alta prevalência de sintomas relacionados à baixa ingestão alimentar, à constipação intestinal e às náuseas ${ }^{14}$, sendo que 20,5\% dos pacientes relataram inapetência; $19,5 \%$ constipação intestinal; 13,9\% náuseas e 12,8\% saciedade precoce ${ }^{15}$. Para medir, analisar e interpretar reações e aceitabilidade dos alimentos e preparações modificados, ou não, deve-se fazer uso da análise sensorial ${ }^{13}$. O estudo avaliou a aceitação de preparações e sua associação com os sintomas decorrentes do tratamento em pacientes de uma clínica especializada em tratamento oncológico.

\section{MATERIAIS E MÉTODOS}

\section{Caracterização da população}

Trata-se de um estudo transversal, descritivo e quantitativo, realizado em uma clínica especializada em tratamento oncológico durante os meses de agosto e setembro de 2011. Para caracterização da população, utilizou-se um questionário desenvolvido no local contendo informações pessoais, dados clínicos, tipo de câncer e tratamentos, além de uma relação dos sintomas frequentes nessa patologia condizente com a Avaliação Subjetiva Global Produzida pelo Paciente (ASG-PPP) ${ }^{16}$. Para aferição do peso, o paciente ficou em pé no centro da balança, descalço e com roupas leves; para a estatura, o mesmo foi posicionado mais próximo do estadiômetro com os calcanhares juntos, costas retas e braços estendidos ao longo do corpo ${ }^{17}$. Os valores de peso e estatura foram utilizados para calcular o índice de massa corpórea (IMC), classificado segundo os critérios da Organização Mundial da Saúde (OMS) para adultos e da Organização Panamericana de Saúde (OPAS) para indivíduos maiores de 60 anos $^{18,19}$, sendo que sobrepeso e graus de obesidade dos adultos foram agrupados na categoria 'excesso de peso. Dentre os dados clínicos, o tratamento cirúrgico foi considerado atual quando realizado em até um ano anterior ao preenchimento do questionário.

\section{Análise sensorial}

As três preparações utilizadas para análise sensorial foram determinadas com base na prevalência dos sintomas descritos ${ }^{14,15}$ e selecionadas a partir de materiais desenvolvidos previamente pelo Serviço de Nutrição do local. Visando à regulação do funcionamento intestinal, foi eleito o tabule havaiano devido ao seu alto teor de fibras. O bolo de gengibre com linhaça, por ser uma preparação hipercalórica e conter gengibre, foi destinado a pacientes com sintomas de baixa aceitação alimentar e náuseas. Igualmente, com o intuito de atingir sujeitos que apresentam inapetência e saciedade precoce, escolheu-se uma preparação com alto teor proteico, incluindo leite em pó, hidrolisado de frango e complemento alimentar (creme Chocolícia). Outros fatores determinantes para a seleção das preparações foram a possibilidade de fazerem parte do hábito dos pacientes, a facilidade do preparo e a mínima utilização de equipamentos como forno e fogão. As receitas foram preparadas no dia da análise sensorial na própria clínica, a qual não possuía tais equipamentos, sendo que as preparações que necessitavam dos mesmos foram elaboradas no dia anterior em outro local, devendo ter assim maior vida de prateleira.

As preparações foram avaliadas por meio do teste afetivo de aceitabilidade com aplicação da escala hedônica de cinco pontos $^{20}$, sendo cinco para gostei muito; três para não gostei nem desgostei; e um para desgostei muito em relação às características de odor, textura, sabor e avaliação global, ou seja, nota sobre a preparação considerando as características organolépticas juntas. Entre as degustações, foi sugerido pela equipe que os participantes ingerissem água para não haver interferência de sabor residual entre as preparações. As preparações foram mantidas sob refrigeração durante a análise, sendo servidas à temperatura em que são usualmente consumidas, em materiais descartáveis de cor branca e em uma mesa coberta com toalha branca. As degustações foram realizadas individualmente em ambiente isolado, tranquilo e isento de ruídos.

\section{Fonte de dados}

As variáveis contidas no questionário de dados pessoais e clínicos estão descritas no Quadro 1 e as questões referentes à avaliação da aceitação das preparações podem ser observadas no Quadro 2. Devido ao uso do hidrolisado de frango em preparação doce, questionou-se sobre a percepção de sabor diferenciado no creme Chocolícia.

\section{Análises estatísticas}

Os dados obtidos do questionário de análise sensorial foram tabulados no Microsoft Excel $^{\varpi} 2007$ (Microsoft Corporation, EUA). As análises estatísticas referentes à aceitação das preparações da população foram realizadas pelo software Fizz 2.3 (Biosysteme ${ }^{\varpi}$, Paris, França). A comparação dos dados de aceitação entre os participantes e em relação às demais variáveis foi realizada no Statistical Package for the Social Sciences ${ }^{\varpi}$ - SPSS 17.0 (SPSS Inc., Chicago, USA), por meio do teste de análise de variância (ANOVA). O nível de confiança adotado foi de $95 \%$.

A receita foi considerada como aceita quando a maior porcentagem $(60,0 \%)$ dos julgadores apresentou resultado de, pelo menos, 'gostei' (nota $\geq 4$ ) na escala apresentada. 


\section{Aspectos éticos}

Os pacientes que se encontravam na clínica foram aleatoriamente convidados e participaram aqueles que assinaram o termo de consentimento livre e esclarecido. A pesquisa teve aprovação do Comitê de Ética em Pesquisa (COEP) da Faculdade de Saúde Pública da Universidade de São Paulo (FSP-USP), protocolo 2.182.

\section{RESULTADOS}

Um total de 23 pacientes participou deste estudo. A idade média da amostra correspondeu a 45,5 $\pm 9,19$ anos, sendo a idade mínima de 38 e a máxima de 87 anos. No total, $43,5 \%$ dos pacientes encontravam-se na faixa etária acima de 60 anos. A média do IMC da população foi de $22,3 \pm 2,96 \mathrm{~kg} / \mathrm{m}^{2}$. As características da população estudada encontram-se na Tabela 1.

Com relação aos dados clínicos, $56,5 \%$ dos casos de câncer primário eram de mama, seguidos por 17,9\% de pulmão. Do total de casos, quatro $(17,4 \%)$ apresentavam metástase à distância, sendo dois com sítio primário em mama e dois em pulmão. Entre os 23 casos, 12 (52,2\%) estavam em tratamento quimioterápico exclusivo, $8(34,8 \%)$ realizaram cirurgia para remoção do tumor em até 12 meses anteriores à coleta de dados em 2011 e $1(4,4 \%)$ estava em radioterapia exclusiva. No momento do estudo, nenhum paciente realizava quimioterapia e radioterapia concomitantemente. Nove (39,1\%) participantes já haviam realizado quimioterapia em algum momento do tratamento, nove $(39,1 \%)$ realizaram cirurgia e seis $(26,1 \%)$, radioterapia. Um total de $39,1 \%$ dos pacientes havia finalizado o tratamento e estava em acompanhamento clínico no momento da coleta de dados.

Aproximadamente $61 \%$ dos pacientes relataram a presença de ao menos um desconforto gastrintestinal nas duas semanas anteriores à coleta de dados, havendo destaque para xerostomia, conforme a Tabela 2. Comparando esses dados com a realização dos tratamentos, observou-se que tratamento quimioterápico atual teve associação estatisticamente significante com a presença de disgeusia $(\mathrm{p}<0,01)$. Para os demais tratamentos e sintomas, não foi observada associação estatisticamente significante (dados não publicados).

Os dados obtidos por meio da escala hedônica da análise sensorial revelaram que as preparações foram aceitas pela maioria da população, conforme Tabela 3 .

Ao provar o creme Chocolícia, $69,6 \%$ dos participantes relataram sentir sabor diferenciado. Desses, três $(18,8 \%)$ não souberam especificar, dois (12,5\%) sentiram certo amargor e nenhum referiu sabor salgado. Os demais sujeitos remeteram ao sabor de oleaginosas, bebidas alcoólicas, adoçantes
Quadro 1. Questionário de dados pessoais e clínicos, São Paulo, 2011

\begin{tabular}{|c|c|}
\hline Variáveis & \\
\hline Dados pessoais & \\
\hline Sexo & Masculino/Feminino \\
\hline Idade & Anos \\
\hline Peso & $\mathrm{kg}$ \\
\hline Altura & $\mathrm{m}$ \\
\hline IMC & $\mathrm{kg} / \mathrm{m}^{2}$ \\
\hline Comorbidades & Câncer \\
\hline & Hipertensão arterial sistêmica \\
\hline & Diabetes mellitus \\
\hline & Hipotireoidismo \\
\hline & Outras \\
\hline Dados clínicos & \\
\hline Tratamento anterior & Quimioterapia \\
\hline & Radioterapia \\
\hline & Cirurgia \\
\hline Tratamento atual & Quimioterapia \\
\hline & Radioterapia \\
\hline & Cirurgia \\
\hline & Não iniciou o tratamento \\
\hline & $\begin{array}{l}\text { Já realizou tratamento e está em } \\
\text { acompanhamento clínico }\end{array}$ \\
\hline Sintomas & Xerostomia \\
\hline & Náusea \\
\hline & Disgeusia \\
\hline & Constipação intestinal \\
\hline & Incômodo com o cheiro da comida \\
\hline & Falta de apetite \\
\hline & Saciedade precoce \\
\hline & Disfagia \\
\hline & Vômito \\
\hline
\end{tabular}

IMC: índice de massa corpórea.

Quadro 2. Ficha de avaliação sensorial

Você receberá três preparações: tabule havaiano, bolo de gengibre com linhaça e creme Chocolícia. Avalie cada preparação quanto ao odor, à textura, ao sabor e global, assinalando com um $\mathrm{X}$ os quadros abaixo e justificando-os.

\begin{tabular}{|c|c|c|c|c|}
\hline Escala hedônica & Odor & Textura & Sabor & $\begin{array}{c}\text { Avaliação } \\
\text { global }\end{array}$ \\
\hline \multicolumn{5}{|l|}{ Desgostei muito } \\
\hline \multicolumn{5}{|l|}{ Desgostei } \\
\hline \multicolumn{5}{|l|}{$\begin{array}{l}\text { Não gostei nem } \\
\text { desgostei }\end{array}$} \\
\hline \multicolumn{5}{|l|}{ Gostei } \\
\hline Gostei muito & & & & \\
\hline
\end{tabular}

dietéticos e outros ingredientes adocicados diferentes daqueles utilizados na preparação.

Comparando pacientes sintomáticos e assintomáticos, houve associação entre saciedade precoce e avaliação global do bolo de gengibre com linhaça $(p=0,049)$, xerostomia e textura do creme Chocolícia $(\mathrm{p}=0,050)$ e disgeusia e avaliação 
global da mesma preparação $(\mathrm{p}=0,045)$. Para os outros sintomas e atributos referentes às preparações, não houve associação significativa (dados não publicados).

\section{DISCUSSÃO}

O perfil etário do presente estudo é semelhante ao de Azevedo e Bosco ${ }^{21}$, que verificaram os perfis nutricional e dietético e avaliaram a qualidade de vida de 20 pacientes em tratamento quimioterápico ambulatorial, no qual a idade média foi de $56,6 \pm 12,7$ anos, com mínima de 30 e máxima de 80 anos, sendo 40,0\% da população acima de 60 anos.

Em relação aos dados antropométricos, a maioria dos pacientes apresentava excesso de peso $(65,2 \%)$, informação também observada por Azevedo e Bosco ${ }^{21}$ em 55,0\% da amostra, cuja média de IMC atual era $28,1 \pm 4,15 \mathrm{~kg} / \mathrm{m}^{2}$. Em outro estudo, $80,0 \%$ dos pacientes que realizavam tratamento quimioterápico em regime ambulatorial apresentavam excesso de peso, havendo prevalência de tumores localizados no sistema gastrintestinal, seguidos do pulmão, mama e ovário, rim e bexiga ${ }^{22}$. Os dados observados no presente estudo podem estar relacionados com a alta prevalência de mulheres e consequente câncer de mama na população, pois excesso de peso é fator de risco para este tipo de neopla$\mathrm{sia}^{23}$. Ademais, um estudo recente envolvendo 175 mulheres sobreviventes do câncer de mama revelou ganho de peso

Tabela 1. Características da população estudada ( $n=23)$, São Paulo, 2011

\begin{tabular}{lcc} 
Variável & $\mathbf{n}$ & $\%$ \\
Sexo & & \\
$\quad$ Feminino & 20 & 87,0 \\
$\quad$ Masculino & 3 & 13,0 \\
Estado nutricional atual & & \\
$\quad$ Baixo peso & 1 & 4,4 \\
$\quad$ Eutrofia & 7 & 30,4 \\
$\quad$ Excesso de peso* & 15 & 65,2 \\
Comorbidades & & \\
$\quad$ Hipertensão arterial sistêmica & 6 & 26,1 \\
$\quad$ Diabetes mellitus & 2 & 8,7 \\
Hipotireoidismo & 2 & 8,7 \\
$\quad$ Outras & 2 & 8,7 \\
\hline
\end{tabular}

*agrupamento de sobrepeso e graus de obesidade em pacientes menores de 60 anos.

Tabela 3. Média e desvio padrão das notas dadas pelos julgadores de acordo com os atributos analisados e a porcentagem de aceitação das preparações analisadas, São Paulo, 2011

\begin{tabular}{lccccc} 
& Odor & Textura & Sabor & Avaliação global & $\begin{array}{c}\text { \% Aceitação } \\
(\text { nota } \geq 4 \text { em \%) }\end{array}$ \\
\cline { 2 - 5 } $\begin{array}{l}\text { Tabule havaiano } \\
\text { Bolo de gengibre }\end{array}$ & $3,50(1,38)$ & $3,67(1,37)$ & $3,50(1,38)$ & $3,67(1,37)$ & 82,6 \\
$\begin{array}{l}\text { com linhaça } \\
\text { Creme Chocolícia }\end{array}$ & $4,50(0,55)$ & $4,50(0,55)$ & $4,50(0,55)$ & $4,50(0,55)$ & 87,0 \\
\hline
\end{tabular}

*teste ANOVA; DP: desvio padrão.

posterior ao diagnóstico da doença em $71,4 \%$ da amostra, independentemente da faixa etária ${ }^{24}$. Tal ganho pode estar relacionado com ingestão alimentar, redução da atividade física, alteração da taxa metabólica basal, menopausa, tratamento antineoplásico (uso de corticoides e terapia hormonal) e aumento de mediadores inflamatórios (como citocinas), podendo ocasionar degradação proteica, expansão de líquido extracelular e consequente retenção hídrica, mascarando o estado nutricional real ${ }^{25}$.

Menos da metade dos pacientes possuía comorbidades associadas (39,0\%), com destaque para hipertensão arterial sistêmica (HAS), em 26,1\%. Azevedo e Bosco ${ }^{21}$ relataram a presença de comorbidades em $55,0 \%$ da amostra de pacientes e, igualmente, maior proporção de HAS entre eles (25,0\%).

Os tumores primários de maior incidência foram os de mama e pulmão, corroborando os dados da estimativa para 2010 e 2011 do Instituto Nacional do Câncer (Inca) ${ }^{3}$. O aumento da incidência do câncer de mama está relacionado à crescente prevalência da exposição aos seus fatores de risco, devido ao intenso processo de urbanização, com aumento do consumo de alimentos industrializados, redução da atividade física e mudanças no comportamento reprodutivo, tais como nuliparidade e idade avançada na primeira gestação ${ }^{23}$. A prevalência de metástase $(17,4 \%)$ foi semelhante ao estudo de Ulsenheimer et al. ${ }^{22}$, que revelou $22,2 \%$, e menor que o de Azevedo e Bosco ${ }^{21}$, que indicou 75,0\% do total da amostra.

Tabela 2. Prevalência de sintomas presentes nas duas semanas anteriores à coleta de dados, relatados pelos pacientes $(n=23)$, São Paulo, 2011

\begin{tabular}{llc} 
Sintoma & n & $\%$ \\
Xerostomia & 7 & 30,4 \\
Náusea & 6 & 26,1 \\
Disgeusia & 6 & 26,1 \\
Constipação intestinal & 6 & 26,1 \\
Incômodo com o cheiro da comida & 6 & 26,1 \\
Falta de apetite & 4 & 17,4 \\
Saciedade precoce & 3 & 13,0 \\
Disfagia & 3 & 13,0 \\
Vômito & 3 & 13,0 \\
\hline
\end{tabular}


A disponibilidade exclusiva da realização de atendimento clínico e de quimioterapia no local pode ter induzido à prevalência deste tipo de tratamento $(52,2 \%)$ em relação à radioterapia exclusiva $(4,4 \%)$ e às cirurgias de remoção do tumor $(34,8 \%)$. Dados preliminares envolvendo 1.000 pacientes atendidos em ambulatórios na Itália revelaram que $80,0 \%$ da população realizavam quimioterapia exclusiva no momento da coleta das informações, $10,0 \%$ realizavam radioterapia, $11,0 \%$ haviam passado por cirurgia e $68,0 \%$ realizavam mais de um tipo de tratamento concomitantemente ${ }^{26}$. Contrastando, Paiva et al. ${ }^{27}$ relataram a prevalência de $84,1 \%$ de radioterapia exclusiva, 2,9\% de quimioterapia exclusiva e 13,0\% da combinação de ambas, em um estudo referente às complicações orais decorrentes da terapia antineoplásica em pacientes em regime ambulatorial.

Sintomas decorrentes da própria doença e de seus tratamentos possuem importante relação com a ingestão alimentar e o consequente estado nutricional do paciente ${ }^{28,29}$. Na população de estudo, aproximadamente $61,0 \%$ dos pacientes relataram presença de desconfortos gastrintestinais nas duas semanas anteriores à coleta de dados, porcentagem menor do que aquela relatada por outros estudos semelhantes, de 77,8 a $95,0 \%{ }^{21,22}$. Bozzetti et al. ${ }^{26}$ informaram ainda a prevalência de fadiga $(71,6 \%)$, falta de apetite $(54,3 \%)$, constipação intestinal/diarreia $(50,2 \%)$, saciedade precoce $(41,3 \%)$, náusea/vômito $(39,3 \%)$, disgeusia $(32,9 \%)$ e disfagia/odinofagia $(23,3 \%)$. Paiva et al. ${ }^{27}$ indicaram maior prevalência de xerostomia (79,5\% das complicações agudas e 90,5\% das tardias), seguida de disgeusia (53,8\% das complicações agudas e $23,8 \%$ das tardias) entre as complicações orais de pacientes em terapia antineoplásica. Devido ao reduzido número de pacientes participantes no presente estudo, a prevalência de cada sintoma pode estar subestimada.

Tratamento quimioterápico atual teve associação estatisticamente significante com a presença de disgeusia $(\mathrm{p}<0,01)$, dado também observado em recente revisão sistemática, a qual revelou prevalência de $56,3 \%$ do sintoma em pacientes em quimioterapia exclusiva ${ }^{30}$.

Igualmente, disgeusia foi associada estatisticamente $(\mathrm{p}=0,045)$ com avaliação global da preparação do creme Chocolícia. A atrofia das papilas gustativas e o aumento da viscosidade salivar que ocorrem na disgeusia, decorrente dos tratamentos antineoplásicos, levam à dificuldade de percepção do paladar e da temperatura, originando uma barreira mecânica de saliva espessa que dificulta o contato físico entre a mesma e os alimentos ${ }^{31}$. Pacientes que apresentam esse sintoma podem relatar uma variedade de alterações no sabor dos alimentos, considerando-os mais ou menos salgados, doces, azedos ou amargos, o que afeta negativamente a ingestão alimentar e a qualidade de vida e pode resultar em aver- sões alimentares ${ }^{32}$, devido ao fato de o gosto e o cheiro fornecerem informações sensoriais e prazerosas da alimentação ${ }^{30}$.

A xerostomia, outro possível sintoma das terapias oncológicas, é definida por secura bucal, alteração da superfície da língua, modificações na produção de saliva e na percepção do gosto $^{31,33}$, podendo causar alterações na percepção da textura dos alimentos ${ }^{32,34}$, como evidenciado pela associação encontrada entre ela e a textura do creme Chocolícia $(\mathrm{p}=0,050)$.

Saciedade precoce foi relatada por pacientes com câncer e parece não estar associada ao sítio primário do tumor, à radioterapia ou à quimioterapia, podendo ser decorrente de outros sintomas, tais como alterações no gosto e aversões alimentares, de mudanças afetivas ou comprometimentos físicos, como retardo no esvaziamento gástrico. Esse desconforto tem contribuição significativa na redução da ingestão alimentar e na progressão da doença, sendo um indicador independente de prognóstico ${ }^{35}$. A avaliação global do bolo de gengibre com linhaça, preparação rica em gordura, teve associação significante com saciedade precoce $(\mathrm{p}=0,049)$.

Segundo Blundell et al. ${ }^{36}$, a gordura possui pouco efeito na saciação (satisfação que ocorre durante a refeição e que inibe a fome, ou seja, é uma resposta imediata), apesar de tê-lo na saciedade (satisfação que ocorre após a ingestão e que dura até a próxima refeição, portanto é uma resposta tardia) por retardar o esvaziamento gástrico e estimular a produção do hormônio colecistoquinina ao chegar ao intestino delgado. Como evidenciado no presente estudo, em alguns indivíduos a ingestão de preparações ricas em gordura pode causar resposta imediata e, consequentemente, redução na ingestão.

A utilização de hidrolisado proteico em preparações dietéticas é recomendada para indivíduos que apresentam necessidades nutricionais e/ou fisiológicas dificilmente cobertas pela alimentação convencional, devido à maior absorção das proteínas em estado cindido ${ }^{37,38}$. O hidrolisado proteico pode fornecer sabor amargo residual, resultante da presença de aminoácidos livres ${ }^{38,39}$, que deve ser mascarado, eliminado ou reduzido visando à aceitação da preparação $0^{38}$. Em um estudo de análise sensorial de preparações contendo hidrolisado de carne, frango ou peru, cerca de $5 \%$ dos participantes referiu sabor amargo residual, que é um valor considerado baixo ${ }^{40}$. No presente estudo, $12,5 \%$ dos participantes sentiram sabor amargo e nenhum deles sentiu o salgado, provavelmente resultantes do hidrolisado, ao degustar a preparação do creme Chocolícia, porém tal número é considerado baixo ao verificar boa aceitação da preparação (78,3\%).

Dados da análise sensorial revelaram aceitação de mais de $78,0 \%$ dos pacientes para todas as preparações, contrastando com o estudo de Azevedo e Bosco ${ }^{21}$, em que 70,0\% dos pacientes relataram aceitação regular ou ruim da dieta. 
Como limitação deste estudo, destaca-se que a avaliação do estado nutricional foi realizada unicamente pelo IMC, dado limitado por não estimar as proporções de reservas muscular e adiposa. A utilização dos parâmetros nutricionais em conjunto contribui para a avaliação do real estado nutricional dos pacientes e a eficiência do tratamento ${ }^{25}$. A bioimpedância elétrica e a aferição de dobras cutâneas podem complementar a avaliação do diagnóstico, ao levar em conta as alterações metabólicas do paciente oncológico e a possível retenção hídrica originada por diversos fatores ${ }^{25}$. Além disso, não foi realizada análise da porcentagem de perda de peso não intencional, fato que podia indicar perda de massa muscular e estar mascarado pela classificação de eutrofia segundo IMC, associando-se a um pior desfecho da doença e à piora da qualidade de vida e da sobrevida ${ }^{22}$.

\section{CONCLUSÕES}

Houve aceitação das preparações, sendo que os sintomas saciedade precoce, xerostomia e disgeusia interferiram em algumas delas na avaliação global da mesma preparação, mas não resultou em sua recusa. A elaboração de receitas criativas e palatáveis a essa população recupera o prazer ao se alimentar, promovendo maior qualidade de vida e melhorando o convívio social. A fisiopatologia do câncer causa alterações metabólicas importantes, as quais são agravadas pelos efeitos decorrentes dos tratamentos. É função do nutricionista minimizar tais desconfortos, conservar e recuperar o estado nutricional e o sistema imunológico do paciente por meio da adequação quantitativa e qualitativa da dieta, levando em consideração aspectos sociais, psíquicos e culturais.

Há, porém, necessidade de mais estudos sobre o perfil dos pacientes oncológicos atendidos em regime ambulatorial e a aceitação de preparações por meio da análise sensorial com essa população, a fim de entender suas reais necessidades e aprimorar técnicas dietéticas e de aconselhamento nutricional.

\section{AGRADECIMENTOS}

Ao Instituto Paulista de Cancerologia e à Clínica SainteMarie pelo espaço fornecido para realizar a análise sensorial e as preparações culinárias, assim como aos pacientes que aceitaram participar voluntariamente do estudo.

\section{REFERÊNCIAS}

1. Instituto Nacional do Câncer [Internet]. O que é câncer. s/a. [cited 2011 Aug 28]. Available from: http://www2.inca.gov.br/wps/wcm/connect/ cancer/site/oquee/

2. World Health Organization [Internet]. International Agency for Research on Cancer. World Cancer Report 2008. França. [cited 2011 Sept 01]. Available from: http://www.iarc.fr/en/publications/pdfs-online/ wcr/2008/index.php

3. Instituto Nacional do Câncer. Estimativa 2010: incidência de câncer no Brasil. Rio de Janeiro: INCA; 2009.

4. Oliveira T. A importância do acompanhamento nutricional para pacientes com câncer. Rev Prática Hosp. 2007;51:150-4.

5. Instituto Nacional do Câncer. Ações de enfermagem para o controle do câncer: uma proposta de integração ensino-serviço. 3. ed. Rio de Janeiro: INCA; 2008.

6. Toscano BAF, Coelho MS, Abreu HB, Logrado MHG, Fortes RC. Câncer: implicações nutricionais. Comunic Cienc Saúde. 2008;19(2):171-80.

7. Silva MPN. Síndrome da anorexia-caquexia em portadores de câncer. Rev Bras Cancerol. 2006;52(1):59-77.

8. Instituto Nacional do Câncer. Controle de sintomas do câncer avançado em adultos. Rev Bras Cancerol. 2000;46(3):243-56.

9. Picazo AM. Náuseas y vómitos. Enfermería Oncol. 2006;2(3):66-70.

10. Ryan JL, Heckler C, Dakhil SR, Kirshner J, Flynn PJ, Hickok JT, Morrow GR. Ginger for chemotherapy-related nausea in cancer patients: a URCC CCOP randomized, double-blind, placebo-controlled clinical trial of 644 cancer patients. J Clin Oncol. 2009;Suppl 15:27.
11. Pillai AK, Sharma KK, Gupta YK, Bakhshi S. Anti-emetic effect of ginger powder versus placebo as an add-on therapy in children and young adults receiving high emetogenic chemotherapy. Pediatr Blood Cancer. 2011;56:234-8

12. Caponero R, Jorge JMN, Melo AGC. Consenso brasileiro de constipação intestinal induzida por opióides. Rev Bras Cuidados Paliativos. 2009;Suppl 1:1-34.

13. Elman I, Pinto e Silva MEM. Crianças portadoras de leucemia linfoide aguda: análise dos limiares de detecção dos gostos básicos. Rev Bras Cancerol. 2007;53(3):297-303

14. Carreiro ABC, Castro NP, Buschinelli LKO, Pinto e Silva MEM. Avaliação da alimentação de pacientes com câncer [trabalho de conclusão]. São Paulo (SP): Faculdade de Saúde Pública da Universidade de São Paulo; 2010.

15. González AT, Prieto JMI, Buschinelli LKO, Philippi ST. Elaboração de preparações fontes de fibra alimentar para pacientes oncológicos com sintomas de constipação intestinal [trabalho de conclusão]. São Paulo: Faculdade de Saúde Pública da Universidade de São Paulo; 2010.

16. Bauer J, Capra S, Ferguson M. Use of the scored Patient-Generated Subjective Global Assessment (PG-SGA) as a nutrition assessment tool in patients with cancer. Eur J Clin Nutr. 2002;56:779-85.

17. Lohman TG, Roche AF, Martorell R. Anthropometric standardization reference manual. Champaign: Human Kinetics Books; 1988.

18. World Health Organization. Diet, nutrition and the prevention of chronic diseases: report of a joint WHO/FAO expert consultation. Geneva; 2003. WHO technical report series, 916. 
19. Organização Pan-Americana de Saúde [Internet]. Encuesta multicêntrica: salud bienestar y envejecimiento (SABE) en América Latina y el Caribe. Washington (DC); 2001 Informe Preliminar. [cited 2011 Oct 12]. Available from: http://www.opas.org/program/ sabe.htm

20. Faria EV, Yotsuyanagi K. Técnicas de análise sensorial. 2. ed. Campinas: Instituto de Tecnologia de Alimentos/Laboratório de Análises Físicas, Sensoriais e Estatísticas; 2008.

21. Azevedo CD, Bosco SMD. Perfil nutricional, dietético e qualidade de vida de pacientes em tratamento quimioterápico. ConScientiae Saúde. 2011;10(1):23-30.

22. Ulsenheimer A, Silva ACP, Fortuna FV. Perfil nutricional de pacientes com câncer segundo diferentes indicadores de avaliação. Rev Bras Nutr Clin. 2007;22(4):292-7.

23. Garófolo A, Avesani CM, Camargo KG, Barros ME, Silva SRJ, Taddei JAAC, Sigulem DM. Dieta e Câncer: um enfoque epidemiológico. Rev Nutr. 2004;17(4):491-505.

24. Rubin BA, Stein AT, Zelmanowicz AM, Rosa DD. Perfil antropométrico e conhecimento nutricional de mulheres sobreviventes de câncer de mama do sul do Brasil. Rev Bras Cancerol. 2010;56(3):303-9.

25. Tartari RF, Busnello FM, Nunes CHA. Perfil nutricional de pacientes em tratamento quimioterápico em um ambulatório especializado em quimioterapia. Rev Bras Cancerol. 2010;56(1):43-50.

26. Bozzetti F. Screening the nutritional status in oncology: a preliminary report on 1.000 outpatients. Support Care Cancer. 2009;17:279-84.

27. Paiva MDEB, Moraes JJC, Biase RCCG, Batista OM, Honorato MCTM. Estudo retrospectivo das complicações orais decorrentes da terapia antineoplásica em pacientes do Hospital Napoleão Laureano - PB. Odontol Clin Cient. 2007;6(1):51-5.

28. Silva PB, Lopes M, Trindade LCT, Yamanouchi CN. Controle dos sintomas e intervenção nutricional: fatores que interferem na qualidade de vida de pacientes oncológicos em cuidados paliativos. Rev Dor São Paulo. 2010;11(4):282-8.

29. Rolim PM, Souza KM, Figueira LP, Silvia LC. Apresentação da refeição versus desperdício de alimentos na alimentação de pacientes on cológicos. Alim Nutr Araraquara. 2011;22(1):137-42.
30. Hovan AJ, Williams PM, Stevenson-Moore P, Wahlin YB, Ohrn KE, Elting LS, Spijkervet FK, Brennan MT; Dysgeusia Section, Oral Care Study Group, Multinational Association of Supportive Care in Cancer (MASCC)/International Society of Oral Oncology (ISOO). A systematic review of dysgeusia induced by cancer therapies. Support Care Cancer. 2010;18(8):1081-7.

31. Paiva MDEB, Biase RCCG, Moraes JJC, Ângelo AR, Honorato MCTM. Complicações orais decorrentes da terapia antineoplásica. Arq Odontol. 2010;46(1):48-55

32. Ravasco P. Aspects of taste and compliance in patients with cancer. Eur J Oncol Nurs. 2005;9:S84-S91.

33. Logemann JA, Smith CH, Pauloski BR, Rademaker AW, Lazarus CL, Colangelo LA, Mittal B, MacCracken E, Gaziano J, Stachowiak L, Newman LA. Effects of xerostomia on perception and performance of swallow function. Head Neck. 2011;23:317-21.

34. Faramarzi E, Mahdavi R, Mohammad-Zadeh M, Ghaeammaghami J. Incidence of eating problems, taste changes and food preference of cancer patients during radiotherapy. Res J Biol Sciences. 2007;2(5):551-6.

35. Davis MP, Walsh D, Lagman R, Yavuzsen T. Early satiety in cancer patients: a common and important but under recognized symptom. Support Care Cancer. 2006;14:693-8.

36. Blundell JE, Lawton CL, Cotton JR, MacDiarmid JI. Control of human appetite: implications for the intake of dietary fat. Annu Rev Nutr. 1996; 16:285-319.

37. Mira NVM, Marquez UML. Importância do diagnóstico e tratamento da fenilcetonúria. Rev Saude Publica. 2000;34(1):86-96.

38. Pinto e Silva MEM, Mazzilli RN, Barbieri D. Hidrolisado protéico como recurso dietético. J Pediatr (Rio J). 1998;74(3):217-21.

39. Penterich VRA, Von Atzingen MCBC, Pinto e Silva MEM. Determinação da viscosidade e composição nutricional de fórmula infantil à base de hidrolisado proteico de frango. Pediatria (Sao Paulo). 2010;32(2):106-12.

40. Pinto e Silva MEM, Von Atzingen MC. Sensory analysis of hydrolyzed meat preparations. Cienc Tecnol Aliment. 2010;30(2):349-53.

Recebido em: 04/06/2012 Aprovado em: 07/12/2012 